\title{
los combustibles y la combustión en los hornos de cemento*
}

Debo aclarar que, al elegir el tema de la combustión $y$ de los combustibles para des. arrollar en esta Ponencia, no he pretendido abarcar ambas materias en forma general y extensa, sino que, por el contrario, quiero limitarme a exponer concretamente ante u3tedes un caso particular, que ofrece dos facetas distintas: de interés teórico la primera que, por consiguiente, puede ayudar a un mejor conocimiento de los problemas que plantea la combustión, y de interés práctico la segunda, siendo mi deseo el someter a controversia ciertas ideas, confusas a mi modo de ver, sobre los combustibles.

En realidad, el origen de este tema se debe a unas escuetas lineas que aparecen en el libro tan conocido dei famoso y ya fallecido ingeniero alemán Dr. Anselm. Me refiero al libro titulado aLa Fabricación del Cemento Portlandn.

En efecto, en la página 43 de la citada publicación puede leerse textualmente lo siguiente :

«El horno vertical A-K (Anselm-Kock, fábricas de cemento de Perlmoos) es un nuevo sistema de horno vertical, basado en el nuevo método de cocción que signe: Iil aire atmosférico contiene un 21 por $100 \mathrm{de}$ oxígeno, y se encuentra siempre disponible en estado natural. Ahora bien, se en-

(*) Poneacia presentada en los II Coloquios de Diroctores y Técnicos de Fábricas de Cemento, I. T. C. C., marzo 1905. sayó la inyección de oxígeno en un horno vertical (cosa que también podría hacerse en un horns rotatorio), para cocer el crudo, con lo cual se logró un consumo de calor mucho menor ( 760 kcal contra 1.100). Pero, además, se obtuvo un resultado imprevisto, $y$ fué el de ver triplicada la producción. En lugar de producir 100 toneladas, puede producir 300 .

En un principio la mera curiosidad me hizo meditar sobre el contenido de estas lineas, con la única intención de encontrar una explicación lógica a este resultado, con. siderado como totalmente inesperado. Pero las deduciones a las que llegué me produjeron cierta sorpresa, pues resultaba evidente que, no obstante la gran cantidad de investigaciones realizadas sobre los hornos, por lo que se deduce de lo que acabamos de leer, las leyes más fundamentales que regulan la marcha de los mismos permanecen aún ignoradas, ya que el que un investigador de talla $\tan$ indiscutib!e, como la del Dr. Anselm, considerase este aumento de producclón como imprevisto, hace sospechar que en la técnica de los hornos quedan todavía campos inexplorados para la investigación; pues lo imprevisto, tanto en la ciencia como en la técnica, es siempre sinónimo de desconocimiento de las leyes que regulan el curso de los acontecimientos.

Vamos a analizar, por nuestra cuenta y riesgo, la experiencia de Anselm y Kock, 
a fin de tratar de hallar una explicación que justifique este fenómeno de aumento imprevisto de producción.

En el ensayo de Anselm y Kock vemos cómo la única innovación introducida con respecto a los hornos normales es la sustitución del comburente aire por el oxígeno puro, y que las consecuencias o resultados de esta sustitución fueron, por un lado, unct disminución en et consumo específico de calor $y$, por otro, un considerable aumento en el rendimiento de producción.

Fácilmente se comprende que al sustituir el comburente aire por el oxígeno puro, automáticamente se provocan dentro del horno dos fenómenos distintos, aunque en el fondo estén relacionados entre sí:

$1 .^{\circ}$ Se modifica la temperatura de la llama.

$2 .^{\circ}$ Se varía la cantidad de gases de combustión.

Estudiemos el primero de estos fellómenos, es decir, el relativo a la modificación de la temperatura de la llama. Para ello vamos a suponer desde ahora, con el fin de simplificar cálculos, que en el horno vertical, objeto de las experiencias de Anselm y Kock, el combustible empleado fué el "carbono», es decir, un posible carbón puro sin cenizas ni materias volátiles. Pues bìen, si calculamos la temperatura de la llama producida en la combustión de dicho carbono con el aire, se tiene que, teóricamente, ésta se eleva a unos $2.240^{\circ} \mathrm{C}$. Pero si dicho acarbonon lo quemásemos con oxígeno puro, la temperatura de la llama ascendería entonces hasta unos $5.850^{\circ} \mathrm{C}$, debido a que, al eliminarse el nitrógeno que contiene el aire, las calorías del combustible se concentran sobre una masa más reducida de gases.

Piénsese ahora que la clinkerización es una reacción química y que en toda reacción química el factor temperatura influye de manera poderosa en la velocidad de reacción. Por consiguiente, es de presumir que, al clin- kerizar con temperaturas de llama de $5.850^{\circ} \mathrm{C}$, en vez de $2.240^{\circ} \mathrm{C}$, se producirá una economía en el consumo específico de calor, a causa del mayor salto térmico, siendo posiblemente ésta la explicación del resultado del experimento Anselm.Kock en sti aspecto térmico.

Ahora bien, como lo que nosotros estamos tratando de investigar son las causas que motivaron el considerable aumento de producción que experimentó el horno, es natural que determinemos, en primer lugar, la influencia que puede tener la disminución del consumo especifico de calor sobre la producción de un horno, y ver si esta influencia es capaz de explicar la superproducción del horno A-K.

Es bien patente que si en un horno de cemento se puede quemar una cantidad determinada de combustible, la producción de dicho horno dependerá del consumo que requiera la fabricación de una tonelada de clínker; y que, si por cualquier causa dicho consumo disminityera, la producción aumentaría en la misma proporción en que disminnye el consumo especifico de combustible. Por tanto, si en ei horno vertical A-K el consumo de calor por tonelada desciende, la producción del mismo tiene que aumentar. Pero podemos comprobar, sin embargo, que el aumento de producción logrado en el experimento es considerablemente más elevado que lo que cabría esperarse de la reducción conseguida en el consumo específico de calor. En efecto, Anselm manifiesta que el consumo de calor por kilogramo pacó desde $1.100 \mathrm{kcal}$ hasta $760 \mathrm{kcal}, \mathrm{y}$, como la capacidad del horno debe variar en razón inversa del consumu específico de calor, la capacidad de dicho horno debería haber aumentado, como máximo, en un 45 por 100 , mientras que el incremento real de producción logrado fué del 200 por 100 , ya que el horno pasó desde 100 hasta 300 toneladas de producción. 
Vemos, pues, que el aumento de la temperatura de la llama, o, lo que es iguail, la disminución del consumo espeć́fico de calor, no basta para justificar por sí solo el notable aumento del rendimiento del horno $\mathrm{A}-\mathrm{K}$, debiendo existir, por consiguiente, otro factor decisivo, del cual depende la producción de los hornos.

Volvamos, pues, la vista hacia el segundo fenómeno que provoca en los hornos la sustitución deí aire por el oxígeno puro, es decir, hacia la variación de la cantidad de gases de combustión.

Esta variación es muy fácil de apreciar, pues claramente se comprende que, a igualdad de peso de combustible, la combustión con oxígeno puro permite introducir dentro del horno una cantidad de gases mucho más reducida que cuando la combustión se realiza con aire atmosférico, ya que éste contien" un 79 por 100 de nitrógeno inerte.

Automáticamente nos vemos, pues, obligados a relacionar la cantidad de gases de combustión formados en el interior del horno con su rendimiento de producción, $y$, a fin de hallar la clase de relación que existe entre ambos conceptos, analizaremos las mismas cifras, dadas por Anselm, ell el cuadro I de la pág. 20, que comentamos a continuación.

En las columnas I, II y III de este cuadro. aparecen los datos dados por Anselm en su libro «La Fabricación del Cemento Portlandn; por ellos vemos cómo el horno vertical A-K, utilizando aire para la combustión, produce 100 toneladas diarias de clínker, con tun consumo específico de 1:100.100 keal por tonelada, mientras que cuando se emplea ox́geno puro, la producción se eleva hasta 300 toneladas diarias, al mismo tiempo que el consumo específico desciende a 760.000 kcal por tonelada.

Hemos dicho que queríamos relacionar la capacidad del horno con la cantidad de gases formados en la combustión; para ello necẹ- sitamos primeramente conocer ia cantidad de combustible quemado, y este dato nos seria muy fácil deducirlo de las columnas II y III, si supiésemos la potencia calorífica del carbón empleado en el ensayo. Nosotros ignoramos el análisis del combustible que se utilizó, pero este detalle carece de impsr'ancia, pues nada fundamental varía si, como ya dijimos, suponemos, para mayor comodidad nuestra, que se trató de carbono puro de S.100 kcal. Partiendo de este supuesto y efectuando operaciones encontramos, de actuerdo con los datos de las columnas II y III, que el consumo diario de combustible en ambos ensayos se elevó a las cifras que aparecen en la columna IV, en la que vemos cómo en el ensayo con oxígeno puro la cantidad de catbón quemado es algo más dẹl doble que cuando se emplea aire. Conocido $e 1$ peso de carbón y su composición, es muy sencillo calcular, mediante unas elementales ecuaciones químicas, el volumen de gases que se forman en la combustión. Los resultados de este cálcuio son los que aparecen en la columna $V$, comprobándose cómo en la combustión con aire, a pesar de quemarse menos de la mitad de combustible, la cantidad de gases producidos es muchísimo más elevada, debido a que la incorporación de nitrógeno atmosférico es extraordinaria (la incorporación de nitrógeno en la combustión ordinaria representa un aumento en el volumen de los gases formados del 37,6 por 100 ). Sin embargo, hay que tener presente que estos volímenes que aparecen en la columna $\mathrm{V}$ no son reales, porque están referidos a $0^{\circ}$ y $760 \mathrm{~mm}$, mientras que, como hemos visto, las temperaturas de las llamas difieren notablemente en ambos ensayos. Por este motivo, con el fin de calcular los volúmenes efectivos, hemos caiculado previamente en la columna VI las temperaturas de las llamas cuando la combustión se realiza con aire y con oxígeno puro, habiendo determinado, después, con estos datos, mediante las oportunas correc- 
ciones del volumen en función de las temperaturas, los volúmenes efectivos que aparecen en la columna Vil.

Las cifras de la columna VII son muy interesantes, pues vemos cómo en el horno A-K, tanto en la combustión con aire como en la combustión con oxígeno puro, si bien varían las producciones, los consumos especificos de calor, los consumos totales de combustible y las temperaturas en el interior del horno, se mantiene, sin embargo, en ambos ensayos un factor constante: el volumen de los gases que circulan por su interior.

Avancemos aún más y tratemos de investigar cuál es la causa que determina esta igualdad de volúmenes gaseosos que circulan en ambos ensayos.

Hasta el momento presente, por desconocimiento de las leyes de que depende el rendimiento de los hornos, se ha venido adop. tando el criterio de expresar éste cmpiricamente, refiriéndolo al volumen de los mismos, según cifras deducidas de la experiencia, y así vemos que se habla de producciones especificas, en toneladas por metro cúbico de horno y día. $\mathrm{Y}$ se comprueba que, realmente, las producciones de los hornos corrientes o normales se obtienen de dicho volumen de los hornos, multiplicando por un cierto factor.

Esto quiere decir que, por dcpender del volumen, de lo que realmente dcpenden las producciones de los hornos es de su sección $y$ de su longitud. Puede, pues, escribirse que:

$$
\text { producción }=(\mathbf{S} \times \mathbf{L}) f \text {. }
$$

Pero, como por otro lado es también evidente que la producción depende igualmente de la cantidad de combustible que puede ser quemada; dividida por la cantidad que de dicho combustible se precisa para la obtención de una tonelada de clínker (consumo específico), o bien multiplicada por la cantidad de clinker que puede fabricarse con una tonelada de carbón (producción específica), puede igualinente escribirse que:

$$
\text { proctucción }=0 \times p_{\iota}
$$

luego no hay inconveniente en admitir, ante la semejanza de estas dos expresiones, que:

$$
(S \times L) f=Q \times p_{e},
$$

siendo necesario reconocer que:

$$
\frac{S \times f}{Q}=-p_{\mathrm{L}}
$$

es decir, que ia cantidad de carbón que puede ser quemada en un horno es función de su diámetro, mientras que la cantidad de calor necesaria para fabricar una tonelada de clinker, a igualdad de circunstancias, es funciin de la longitud. Por este motivo, dcs hornos de igual diámetro y distinta longitud cleben poder quemar cantidades iguales de combustible, pero sus producciones diferirán porque en el horno más corto los gases calientes están menos tiempo en contacto con el material y escaparán a la atmósfera a elevada temperatura, y, por consiguiente, la producción de la tonelada de clínker exigirá mayor cantidad de calor en detrimento de la capacidad del horno. Por el contrario, en el horno de mayor longitud se eleva el rendimiento térmico a causa del mayor tiempo de permanencia de los gases, aumentando, en consecuencia, el tonelaje de producción.

Admitamos la exactitud del segundo término de esta semejanza, es decir, que la producción específica es función de la longitud del horno, y tratemos de hallar la razón de dependencia entre el diámetro y la cantidad de combustible quemado.

Cuando en un horno se comienza por quemar una cantidad determinada de combustibje, primeramente se regula adecuadamente el registro de la chimenea, de forma que 
en el horno se introduzca la cantidad conveniente de aire para la combustión. La consecuencia de todo ello es la formación de una cantidad de gases a una temperatura determinada. Estos gases ocupan un cierto volumen y adquieren, ell consecuencia, una velocidad que resulta ser función de la sección del horno y del volumen gaseoso formado. Cuando se aumenta la cantidad de combustible inyectado, se hace necesario manipular nuevamente en el registro de tiro para aumentar proporcionalmente lá e:Iírada de aire y, como es natural, el volumen gaseoso formado crece, viéndose los gáses ob'igados, en consecuencia, a aumentar su velocidad de circtilación. A cada nuevo incremento en el consumo de combustible, sucede siempre análogo fenómeno de aumento de la velocidad de los gases por el horno. Pero esto no ocurre así de manera indefinjua.

Quien ha trabajado con un horno dotado de chimenea sobredimensionada, sabe que existe un limite, ya que, a medida que se aumenta la cantidad de combustible, los gases en un principio comienzan a salir cacla vez más calientes; después hace su aparición el óxido de carbono e, incluso, llames por la chimenea, y más adelante se observa la apa. rición de humo negro procedente del carbón $\sin$ quemar. Este defecto no puede evitarse introducienđo más aire, ya que, al llegarse a este extremo, dicha solución sólo sirve para agravar aún más el problema; es decir, que lo que no puede aumentarse indefinidamente dentro del horno es la velocidad de los gases, 0, lo que es 10 mismo, que existe una velocidad limite de circulación gaseosa y que, al sobrepasarla, la combustión no se efectúa ya correctamente.

Ya tenemos, pues, la explicación de la igualdad de los volúmenes gaseosos que circulan en los dos ensayos de combustión realizados en el horno A-K con aire $y$ con oxígeno, y la interpretación del aumento de producción considerado por Anselm como imprevisto es ahora muy sencilla.

En efecto, ya hemos dicho que existe un limite para la velocidad de circulación gaseosa, y precisamente por este motivo vemos en la collumna VII cómo en ambos ensayos los volúmenes gaseosos efectivos quc circulan por el horno son prácticamente iguales. Ahora bien, tn el caso de la combustión con aire, el 79 por $\mathbf{1 0 0}$ del volumen es nitrógeno inerte (la nisma proporción que en el aire, ya que un mol de $\mathrm{O}_{2}$ produce un mol de $\mathrm{CO}_{2}$ que ocupa el mismo volumeni). De ello resulta que el volumen de $\mathrm{CO}_{2}$ es casi cinco veces inferior al volumen del mismo gas producido cuando la combustión se efectía con oxígeno. Pero estos volúmenes no $\$>17 \mathrm{com}$ parativos porque están a diferentes temperaturas. Llevándolos a las condiciones normales de presión y temperatura, vemos en la columna $\mathrm{V}$ cómo en realidad la cantidad de $\mathrm{CO}_{2}$ en la combustión con oxigeno es algo más del doble que en la combustión con aire, $\mathrm{y}$, como el anhídrido carbónico es el resultađo de la reacción de combustión, las cantidades de carbón quemadas son proporcionales a estos volúmenes normales de $\mathrm{CO}_{2}$. como puede verse en la columna IV, en que la cantidad de carbón quemada con oxígeno es también algo más del doble. Por este motivo, y también porque al mismo tiempo el consumo específico de calor es menor, la producción del horno vertical A-K queda triplicada cuando se empleó oxígeno como comburente.

Resumiendo, podemos decir que, cuando en el horno vertical A-K se utiliza oxígeno como comburente, su producción se triplica:

1. Por una disminución del consumo específico de calor a causa de la mayor temperatura de la llama.

2.' Porque puede aumentarse la cantidad de combustible quemado hasta un límite en el que el nuevo volumen efectivo de anhídrido carbónico formado sea igual al volumen 
de nittógeno atmosférico qtie deja de introcirse, de forma que, en último extremo, los rolúmenes gaseosos que circuan por el horno son los mismos.

Restaría ya solamente determinar por qué existe dicha velocidad límite en los gases, a la cual queda supeditada ín cantidad máxima de combustible que puede ser quemad? en los hornos y, en consecuencia, la producción de los nismos. Es muy posible que esta limitación esté relacionada con la veloc:dad de reacción entre combustible y comburente : porque es irdudable que ia combustión exige un cierta tiempo para realizarse, y, si el combustible se desplaza dentro del horno más rápidamente que su velocidad de reacción, ésta quedará incompleta por falta cle tiempo para verificarse.

Pero cuanto hemos dicho es válido, no solamente para el horno vertical A-K, sino para cualquier tipo de horno. Colsviene, por consiguiente, que dejemos clarrmente expuestas las deducciones generales al lis que hemos llegado, y que escuetamente son las siguientes :

1. La cantidad de carbón que puecte quemarse en un horno $\mathrm{y}$, en consecuencia, su producción, está limitada por una ds"erminada velocidad máxima de los gases que circulan por su interior.

2. Esta limitación de la velocidad depende muy probablemente de una ciertá relación entre la velocidad de circulación gaseosa y la velocidad de combustión.

3. La cantidad de catbón que puede quemarse, es decir, la producción de un horno, estará influenciada: por la naturaleza química y física del combustible, por el modo de realizarse la combustión y por el combinrente empleado, pues de todos estos factores depende la cantidad y temperatura de los gases formados, es decir, el volumen del que, a su vez, es función la velocidad de circulación.
4. Por tanto, a igualdad de las anteriores condiciones, la producción de los hornos será proporcional al cuadrado del diámetro del horno $e$ inversamente proporcional al consumo específico de calor, que dependerá, a str vez, de la longitud y de la composición quimica del material crudo.

5. Consecuencia de todo lo dicho es que la constante de un horno es su capacidad de circulación gaseosa, mientras que los restantes factores (producciones, temperaturas, etc.) son variables que dependen del modo de realizarse ia combustión.

Las deducciones que pueden extraerse de estos principios fundamentales son muy numerosas, abarcando considerable número de problemas sobre la combustión, sobre los diferentes tipos o sistemas de hornos y sobre los combustibles. Sin embargo, con el fin de no extendernos demésiado, vilmos a limitarnos a tratar únicamente de los combustibles, los cuales, por su importancia económica, constituyen materia de extracrdinario interés en nuestra industria.

Al dar comienzo a mi disertación dije que mi intención era el someter a controversia ciertas ideas, confusas, a mi modo cle ver, sobre los combustibles. Concretamente voy il referirme a lo que se ha dado en liamar acarbones de baja calidadn. La importancia de este tema es desde luego grande, y creo que fué la Agrupación de Fabricantes, la que, ante el problema creado por la stistitución de los carbones clásicos, de elevada potencia calorífica, que se empleaban en nuestra industria con anterioridad a nuestra Cruzada de Liberación, por carbones de alto contenido de cenizas, consideró interesante convocar y premiar, hace ya unos cuantos an̄os, aquellos trabajos que sirvieran para orientar sobre el empleo de dichos carbones y eliminar, et lo posible, los funestos efectos que se les atribuye sobre las producciones $y$ las calidades del cemento fabricado con ellos, Y tan grande es el perjuicio que oca- 
sionan dichos carbones, que ha habido quienes han considerado necesario, con motivo de los convenios hispano-americanos, la inportación de carbones selectos, a fin de poder lograr obtener calidades de cemento que cumplieran con las Normas de los Estados Unidos.

Yo voy a salir un poco en defensa de los tan vilipendiados carbones de baja calidad, porque considero que el asunto tiene un gran interés económico, desde el momento en que dichos carbones son mucho más baratos que los carbones buenos, y porque, según mi parecer, puteden llegar a ser empleados con éxito, siempre que se estudie este problema con detenimiento. Pues la verdad es que, no obstante la extraordinaria repercusión económica que sobre el precio de coste de fabricación representa el capítulo de combustión, ya que por sí solo se eleva a cerca del 50 por $\mathbf{1 0 0}$ de su importe total, lo que debiera haber obligado a conceder la máxima importancia a los problemas que plantea su rendimiento, en realidad se tienen un poco en olvido dichos problemas, llegándose a confundir conceptos muy diferentes. Si, por ejemplo, yo manifiesto aquí que la combustión de un carbón con $11 n$ so por 100 de cenizas, es decir, con unas $4.000 \mathrm{kcal}$, aproximadamente, debe proporcionar tuna temperatura tan elevada como un "fuel-oil» de $10.000 \mathrm{kcal}$, es posible que haya quienes duden o nieguen esta afirmación.

Para poder entrar en materia, es necesario que previamente revisemos unos cuantos conceptos fundamentales sobre la combustión.

La combustión, como es bien sabido, es una reacción química de oxidación realizada entre los elementos combustibles y el oxígeno del aire. Esta reacción es fuertemente exotérmica, es decir, que se verifica con desprendimiento de grandes cantidades de calor. Los elementos combustibles no entran espontáneamente en reacçión con el oxígeno del aire, sino que para que la reacción se inicie es necesario llegar a la temperatura de inflamación. A esta temperatura se desprenden elementos volátiles que arden con producción de calor, el cual eleva aún más Ia temperatura del combustible, por lo que la combustión prosigue ya por sí sola, alcanzándose entonces la temperatura de combustión.

Los combustibles pueden ser sólidos, liquidos y gaseosos. Hasta hace poco tiempo en Españat sc empleaban exclusivamente combustibles sólidos, pero últimamente se va generalizando el empleo de los combustibles licpuidos.

Como la combustión es una reacción química, st estudio exige el conocimiento del análisis químico elemental del combustible. El análisis químico de un combustible, tanto sólido como líquido, pone de manifiesto que, en todos los casos, los elementos combustibles se reducen al carbono, al hidrógeno y al azufre, acompañados siempre de ciertas cantidades de oxígeno, que también interviene en las reacciones, además de otros cuerpos que no desempeñan ningún papel directo en las mismas (cenizas, humedad, nitrógeno).

Las consecuencias inmediatas de las reac. ciones de combustión son dos:

1.* Producción de calor.

2, Formación de una cantidad de gases de combustión,

Pero para la industria del cemento el problema de los combustibles adquiere, por lo que se desprende de cuanto hemos dicho, aspectos muy particulares, porque dependiendo la prođucción de los hornos de una cierta vélocidad límite para los gases que circulan por su interior, el mejor combustible no es aquèl que posee mayor potencia calorífica, sino el que transporta más calorías en un volumen menor de gases y a más elevada temperatura. El cuadro II ayudará a comprender este concepto que acabamos de exponer. 
En este cuadro (II, pág. 20) henos tomado para su estudio los cuatro elementos combustibles que, de una manera $\mathfrak{u}$ otra, están relacionados con la combustión; pues aparte del carbono, que es el elemento básico de cualquier combustible, el azufre casi siempre interviene en dosis variables, aunque siempre reducidas, y ell hidrógeno se halla siempre presente en cantidades que varían según el contenido de volátiles, y que van desde el 2 por 100 en los carbones muy antracitosos, hasta el 12 por 100 en el fuel-oil.

En ta columna II de este cuadro se tiene la potencia calorífica de cada uno de estos combustibles, viéndose cómo ésta es muy reducida para el óxido de carbono y para el azufre con, aproximadamente, unas $\mathbf{2 . 3 0 0}$ calorías por kilogramo, y considerablemente elevada, unas 34.200 kcal para el hidrógeno, si bien la potencia calorífica inferior de este elemento es bastante más reducida, 28.700 calorías, a consecuencia del agua formada en la combustión del mismo. Para el carbono ta potencia calorifica es de 8.100 calorías.

En la columna III hemos calculado los volúmenes gaseosos formados en las distintas combustiones, a consecuencia de la combinación de los elementos combustibles con el oxígeno del aire, comprobándose cómo estos volúmenes varían extraordinariamente, pues oscilan desde $2 \mathrm{~m}^{3}$ para el óxido de carbono, hasta $32 \mathrm{~m}^{\mathrm{g}}$ para el hidrógeno.

En la columna IV se tiene la explicación de esta variación tan extraordinaria en 105 volúmenes formados, pues puede verse cómo la cantidad de oxígeno que precisa cada reacción de combustión varía también considerablemente, $y$ hay que tener presente que en el proceso de combustión se incorpora a los humos una gran cantidad de nitrógeno contenido por el aire y que, por tanto, el volumen de gases será tanto mayor cuanto más oxígeno se requiera. Por este motivo el hidrógena forma un volumen 16 veces mayor quie el óxido de carbono.
En la colımna $V$ está callculada la temperatura de las llamas formadas en cada combustión, viéndose que estas temperaturas no guardan ninguna relación con la potencia calorífica del combustible, ya que la temperatura, no solamente es función de la cantidad de calor desarrollado, sino también del volumen gaseoso formado. A mayor cantidad de gases, menor temperatura, porque el calor queda diluido en una mayor masa gaseosa.

Como ya dijimos que la producción de un horno es función de la velocidad y ésta, a su vez, to es del volumen efectivo de los gases, en la columna VI, mediante las oportunas correcciones volumétricas y de acuerdo con las temperaturas respectivas, hemos calculado los volúmenes efectivos de las correspondientes combustiones.

$Y$, por último, en la columna VII, teniendo en cuenta la potencia calorífica y los volúmenes efectivos, hemos hallado, para cada combustión, las calorias transportadas por metro cúbico. Por tanto, para una velocidad dada de corriente gaseosa, o, lo que es lo mismo, para un volumen constante de gases, la producción de an horno será tanto mayor cuanto mayor sea la cantidad de calor introducido. Vemos, pues, que de estos cuatro combustibles, el óptimo no sería precisamente el hidrógeno con sus $34.200 \mathrm{cal}$, ni siquiera el carbono con sus 8.100 calorias, sino el óxido de carbono que sólo posee $\mathbf{2 . 4 4 0}$ cal, debido al menor volumen de gases que se forma en su combustión. El empleo de este combustible aumentaría aproximadamente el rendimiento de un horno en un 20 por 100 , si se tiene en ctenta que, además, es el que proporciona una temperatura más elevada. En cuanto al carbono y al hidrógeno, por igual causa, el primero viene a ser un combustible de mejor calidad que el segundo. Ei azufre, por el contrario, resulta ser un combustible deficientisimo, muy especial. 
mente por la temperatura de liama tan baja que proporciona.

Ahora es fácil demostrar la afirmación hecha anteriormente de que un carbón con un 50 por 100 de cenizas, es decir, de unas 4.000 calorias, puede proporcionar dentro de un horno una temperatura tan elevada como la de un fuel-oil de $\mathbf{1 0 . 0 0 0}$ calorías, porque, si bien es cierto que cuando tu carbón contiene un 50 por 100 de cenizas produce en su combustión la mitad de calorías que en el caso de estar totalmente exento de cenizas, también es verdad que se formarán la mitad de gases y que, al no existir variación alguna en la relación de calorías a gases formados, sobre los que aquéllas se concentran, las temperaturas no sufrirán tampoco ninguna alteración. Es decir, que las temperaturas de las llamas son independientes del contenido de cenizas de los carbones y dependen sólo de la naturaleza química de los elementos combustibles, y como la única diferencia química que existe entre un carbón y un fuel-oil es que éste último presenta un mayor contenido de hidrógeno, y puesto que, como hemos visto, el carbono y el hidrógeno presentan temperaturas muy similares, se comprende que un fuel-oil de $10.000 \mathrm{cal}$ produzca en stu combustión una temperatura nuy parecidá a la de un carbón, cualquiera que sea su porcentaje de cenizas; esto, por lo menos, desdc el punto de vista teórico. 'Teóricamente el límite en el contenido de cenizas dele hallarse en aquel punto en que el carbón necesite para su inyección dentro del horno una cantidad de aire superior a la necesaria para la combustión de sus elementos combustibles, en cuyo caso la temperaturi disminuiría a causa del exceso de aire.

La producción de un horno no debería, pues, resultar afectada por el empleo de carbones de baja calidad, ya que, a primera vista, todo el problema se reduce a compensar el exceso de cenizas del carbón con un au- mento en el consumo, de forma que en cualquier caso la cantidad real de verdaderos elementos combustible: inyectados en el horno permanezca invariable. Ls decir, que al introducir, independientemente del contenido de cenizas, lia misma cantidad de combustible real, el calor producido será el mismo, la cantidad de gases formados tampoco variará, y la temperatura se mantendrá idénticamente la misma, y, por tanto, no existe motivo para que la producción disminuya.

Sin embargo, la verdad es que en la práctica industrial el uso de carbones de elevado contenido de cenizas no concuerda con las deducciones teóricas a que hemos llegado, y que su empleo se traduce normalmente en combustiones defectuosas con mermas en las producciones, $y$ en calidades deficientes de los cementos, y que, por consiguiente, los directores de fábrica, prefieren adquirir carbones buenos, aunque con ello, debido a su mayor precio, se encarezca el coste del cemento.

Lo verdaderamente interesante es determinar a qué se debe esta falta de concordancia entre ia teoría y la práctica, y ver si en el orden industrial es posible infiuir favorablemente sobre el proceso de combustión y llegar a utilizar estos carloones con plena satisfacción, de acuerdo con las deducciones teóricas.

Cuando en un horno de cemento se sustituye un carbón de elevada potencia calorífica por otro carbón de elevado contenido de cenizas, se observa que la boca del horno se enfría y la zoná de clinkerización se desplaza hacia el interior $y$, además, esta zona parece menos caliente que cuando se emplean buenos carbones. $\mathrm{Si}$ con el fin de calentar el horno introducimos más carbón, a veces conseguimos sólo sacar humo negro por la chimenea. Por este motivo, la producción desciende $\mathrm{y}$, además, por falta de temperatura, el grado de cocción es bajo y se obtienen calidades deficientes de cementos. 
Todos estos defectos no son debidos a que el elevado contenido de cenizas de un carbón convierta a éste en un producto intrínsecamente poso apto para ser urilizado, sino a que no se tiene en cuenta un aspecto muy importante del problema. En efecto, es bien sabido que en toda reacción duimica en la que intervenga, cuando menos, un cuerpo sólido, el grado de subdivisión desempeña un papel no solamente importante, sino decisivo sobre la velocidad de reacción; y la combustión, que es una reacción química entre un gas, el aire y un sólido-el carbónno escapa a esta regla. Por este motivo, el carbón antes de inyectarlo en los hornos debe ser molido a un grado de finura tan extremo como cualquiera de las otras dos moituraciones que se realizan en nuestra industria (crudo y cemento), ya que, si se moliera muy groseramente, la combustión se realizaría de forma defectuosa, debido, indudablemente, a que se disminuye la velocidad de reacción y, como ya vimos, la cantidad de combustible que puede quemarse en un horno está supeditada a una cierta velocidad de los gases formados que circulan por el horno y, a su vez, esta velocidad de circulación gaseosa debe ser tunción de la velocidad de la reacción de combustiónt.

Cuando se muele el carbón se pretende crear tuna superficie especifica que haga posible la combustión en las condiciones particulares de tiempo y espacio que exige el horno de cemento. El grado de molienda necesario ha sido hallado, en realidad, por la experiencia; y cuando llevamos nuestros carbones, por ejemplo, hasta ul] residuo del 10 por 100 sobre el tamiz de 4.900 mallas, esta finura no es caprichosa, sino la más conveniente, aunque a ella hayamos llegado por el camino de la práctica.

Cuando un carbón contiene cenizas, en realidad disminuye su superficie específica uactivas, porque una parte de la superficje creada no está formada por verdaderos elemen- tos combustibles, sino por las cenizas, que son materias que no intervienen en la combustión, es decir, que sucede como si dicho carbón huibiera sido molicho más groseramente; por este motivo disminuye su velocidad de reacción de combustion $y$, en consecuencia, la combustión se desarrolla anormalmente.

Por esta causa, cuando un carbón contiene un elevacio tanto por ciento de cenizas, si queremos quemarlo bien necesitamos molerIo mucho más finamente para mantener, en lo posible, una superficie específica activa similar a la que presentan los carbones buenos cuando han sido molidos a las finuras corrientes. Yo sé que, en el fondo, esta idea de la necesidad de aumentar el grado de finura de los carbones de baja calidad, sí se quieren utilizar sin inconvenientes, está en la mente de todos los fabricantes, pero hace falta lievar estas ideas hasta el último extremo e investigar cuáles son los grados de finura de molido más convenientes en función del contenido de cenizas de los carbones. Esta es una labor que incumbe a todos, ya que, dada la importancia económicá de este tema, al aclarar esta incógnita de los carbones de baja calidad, habríamos rendido un gran servicio a nuestral industria.

Para terminar, sólo quiero añadir que es muy posible que en mi estudio hayan quedado puntos oscuros, extensas lagunas $y$ tal vez hasta errores, pero piensen ustedes que los que trabajamos en las fábricas resolviendo los diarios y numerosos problemas que se presentan, no somos investigadores que puedan dedicarse a confeccionar impecables teorias, pues para ello no tenemos tiempo ni medios y, posiblemente, ni siquiera capacidad; únicamente en el modesto laboratorio de nuestro cerebro, robando alguila que otra hora al sueño, meditámos sobre la naturaleza de los problemas que se nos ofrecen, $y$ en reuniones tan interesantes como éstas aprovechamos la opor- 
tunidad de exponer nuestras ideas con la única intención de someterlas a controversia y ver si, en provecho de todos, se puede ha- cer un paco más de luz en los rincones que aún quedan por iluminar en nuestra industria.

CUADRO NGS, I

Andisis de los resultados técnicos del Ilorno Vertical $A \cdot K$

\begin{tabular}{|c|c|c|c|c|c|c|c|c|}
\hline \multirow[t]{2}{*}{$\begin{array}{l}\text { Comburante } \\
\text { empleato } \\
\text { en ol ensajo. }\end{array}$} & $\begin{array}{c}\text { II } \\
\text { Producción } \\
\text { ceda } 2 \text { t horas } \\
\text { de marcbs. }\end{array}$ & $\begin{array}{c}\text { JII } \\
\text { Consumo } \\
\text { de tialor en } \\
\text { keal por Tm }\end{array}$ & $\begin{array}{l}\text { lV } \\
\text { Congumo combus- } \\
\text { tible en } 24 \text { horas } \\
\text { expresado en car- } \\
\text { bono de } 8.100 \text { keal. }\end{array}$ & \multicolumn{2}{|c|}{$\begin{array}{l}\text { Volumen de gases } \\
\text { a } 0^{\circ} \text { y } 760 \mathrm{~mm} \text {. }\end{array}$} & $\begin{array}{c}\text { VI } \\
\text { Temperatura } \\
\text { dé la lla ma. }\end{array}$ & \multicolumn{2}{|c|}{$\begin{array}{l}\text { VuI } \\
\text { Volumen efectiro } \\
\text { a la temperatura } \\
\text { correspondiente. }\end{array}$} \\
\hline & $7 \mathrm{~m}$ & do clinker. & $\mathrm{Tm}$ & & $m^{9}$ & ${ }^{\circ} \mathrm{C}$ & & \\
\hline \multirow{2}{*}{ Aire,$\ldots .}$. & \multirow{2}{*}{100} & \multirow{2}{*}{1.100 .000} & \multirow{2}{*}{13,580} & $\begin{aligned} \mathrm{N}_{2} & = \\
\mathrm{CO}_{2} & =\end{aligned}$ & $\begin{array}{l}94.970 \\
25.258\end{array}$ & \multirow{2}{*}{$2.239^{\circ}$} & $\begin{array}{r}\mathrm{N}_{\mathrm{z}}= \\
\mathrm{CO}_{2}=\end{array}$ & $\begin{array}{l}873.790 \\
232.410\end{array}$ \\
\hline & & & & & 120.228 & & & $1.11,6.200$ \\
\hline Oxígeno..... & 300 & 760.000 & 28,140 & $\mathrm{CO}_{2}=$ & 52.340 & $5.852^{\circ}$ & $\mathrm{CO}_{2}=$ & 1.170 .1331 \\
\hline
\end{tabular}

CUA D R O N US. II

Analisis de lo calidad de diferentes combustibles en funcion de las calorias transfortados por $n^{3}$ xe gases efectivos formados.

\begin{tabular}{|c|c|c|c|c|c|c|}
\hline Combustibles. & $\begin{array}{c}\text { II } \\
\text { Potencia } \\
\text { caloriffca del } \\
\text { combustible } \\
\text { keal }\end{array}$ & $\begin{array}{c}\text { 1il } \\
\text { Volúmenes de } \\
\text { gases formados } \\
\text { en 1a combustión } \\
\text { a } 0^{\circ} \text { y } 760 \text {. }\end{array}$ & $\begin{array}{c}\text { IV } \\
\text { Cantidad de oxige- } \\
\text { no necesarjo en } \\
\text { les reacciones } \\
\text { de combustión. }\end{array}$ & $\begin{array}{c}\mathrm{V} \\
\text { Temperaturas } \\
\text { leóricas de } \\
\text { la llama } 0^{\circ} \mathrm{C} .\end{array}$ & $\begin{array}{c}\text { VI } \\
\text { Volúmenes } \\
\text { efectivos de los } \\
\text { gases de combus- } \\
\text { tín a los tempera- } \\
\text { turas teóricas. }\end{array}$ & $\left\{\begin{array}{c}\text { VII } \\
\text { Calorias } \\
\text { transportadas } \\
\text { por } \mathrm{m}^{3} \text { de gases } \\
\text { efecuivas. }\end{array}\right.$ \\
\hline Oxido de carbono... & 2.440 & 2,16 & 0,57 & $2.557^{\circ}$ & 21,7 & 112,4 \\
\hline Carbono......... & 8.100 & 9,00 & 2,96 & $2.243^{\circ}$ & 82.9 & 97,8 \\
\hline Hidrogeno........ & 28.700 & 32,63 & 8,00 & $2.234^{\circ}$ & 300,00 & 95,6 \\
\hline Azufre $\ldots \ldots \ldots \ldots$ & 2.210 & 3,33 & $1,00)$ & $1.958^{\circ}$ & 23,8 & 92.8 \\
\hline
\end{tabular}

